\title{
Development of a Versatile, Full-featured Search Functionality for Indico
}

\author{
Penelope Constanta1,*, Ofer Rind ${ }^{2}$, Jose Caballero Bejar ${ }^{2}$, Pedro Ferreira ${ }^{3}$, Adrian \\ Mönnich $^{3}$, Pablo Panero ${ }^{4}$, Carina Rafaela De Oliveira Antunes ${ }^{5}$, and Aristofanis Chionis \\ Koufakos $^{3}$ \\ ${ }^{1}$ Fermi National Accelerator Laboratory, CCD, P.O. BOX 500, Batavia IL 60510-5011, USA \\ ${ }^{2}$ Brookhaven National Laboratory, Physics Dept., P.O. Box 5000, Upton, NY 11973-5000, USA \\ ${ }^{3}$ CERN, Esplanade des Particules 1, IT-CDA-IC, P.O. Box 1211 Geneva 23, Switzerland \\ ${ }^{4}$ CERN, Esplanade des Particules 1, IT-CDA-DR, P.O. Box 1211 Geneva 23, Switzerland \\ ${ }^{5}$ CERN, Esplanade des Particules 1, IT-CDA-WF, P.O. Box 1211 Geneva 23, Switzerland
}

\begin{abstract}
Indico, CERN's popular open-source tool for event management, is in widespread use among facilities that make up the HEP community. It is extensible through a robust plugin architecture that provides features such as search and video conferencing integration. In 2018, Indico version 2 was released with many notable improvements, but without a full-featured search functionality that could be implemented easily outside of CERN. At both Fermi and Brookhaven National Laboratories, the user community viewed the lack of this popular feature as a significant impediment to deployment of the new software. In the meantime, CERN embarked upon a major redesign of their core search service, one that would also necessitate a rewrite of the Indico search interface. Seeing this pressing need, the two US labs decided to collaborate, with assistance from the CERN development team, on a project to develop the requisite search functionality for the larger user community. The resulting design exploits the simplified schema defined in the new CERN Search micro-service, based on Invenio and Elasticsearch, while still providing a flexible path to implementation for alternative backend search services. It is intended to provide a software package that can be installed easily and used out of the box, by anyone at any site. This paper will discuss the design choices and architectural challenges, and provide an overview of the deployment and use of these new plugins.
\end{abstract}

\section{Overview}

Indico is an open-source event management system, popular in the High Energy Physics (HEP) community. Indico's extensibility through its plugin architecture provides integrated event fee payment (via PayPal), video conferencing, search, room booking etc. that can be modified and/or extended to suit the specific needs of a particular institute. [1][2]

The latest version of Indico v2.x [4][5] has been re-written to use modern Python packages (Flask, SQLAlchemy, marshmallow etc.) and to use PostgreSQL for its database

\footnotetext{
* Corresponding author: penelope@fnal.gov
} 
needs. However, Indico's popular search plugins have not been upgraded and the search functionality is limited to CERN's eco-system that currently uses SharePoint. The necessity for developing new plugins that are not tightly coupled with CERN's eco-system, is twofold: CERN is moving away from SharePoint to the new Invenio based CERN Search microservice; and the Fermi, BNL user communities requested a full functional search before deploying the new version 2.x of Indico.

The Fermi-BNL-CERN collaboration was formed to build the search plugins utilizing the new CERN Search microservice and make them available to the community as an easily deployable solution.

\subsection{Indico's search plugins history}

Indico v0.98 - v1.2: the search plugins utilize Invenio (v1.1) as their search engine. The plugins send search metadata and receive search results using the MARCXML Standards. The framework (Indico and Invenio) are not specific to CERN's infrastructure and can be used outside CERN's environment.

Indico v1.9 - v2.2: CERN itself moved towards a solution which uses an internallyavailable custom solution based on SharePoint, while the officially available Invenio plugin fell into disuse for lack of a maintainer. The CERN solution plugins send search metadata using the MARCXML Standards and search results are displayed by SharePoint.

Future Indico v2.x: the search plugins, as developed in this collaboration effort, will utilize the Invenio's (v3) "CERN Search API" component, built upon Elasticsearch as its search engine. The plugins will send search metadata and receive search results in JSON format. The framework is being developed so that it can be used both internally by CERN as well as outside CERN's environment.

\section{Indico's code architecture}

Indico's application code consists of the core system that provides the main functionalities of the application and the plugin subsystem which extends the application's functionality. The plugins are standalone packages of code that do not require any modifications to the Indico core itself. A plugin can perform something very simple such as adding a new command to the Indico CLI or more complex functionalities like introducing new payment methods, chat integration, etc. [3]

Indico's search functionalities are implemented by a set of plugins. The livesync and search plugins are core Indico plugins that require the implementation of corresponding agent plugins to provide connection with a particular search engine.

Indico's search metadata are collected by the core livesync plugin. Livesync collects metadata from events and their contributions, subcontributions, attachments and notes. The collected metadata are sent to the livesync agent plugin. The livesync agent is responsible for formatting appropriately and transmitting the search metadata to its associated search engine.

Indico's user search interface is implemented by the core search plugin. The search plugin accepts the user's search criteria, sends them to a search agent plugin and then receives and displays the search results. The search agent plugin is responsible for formatting and transmitting the search criteria as well as receiving the search results and 
sending them to the core search plugin. The Indico search architecture and the Fermi-BNLCERN effort architecture are illustrated in Figures 1 and 2.

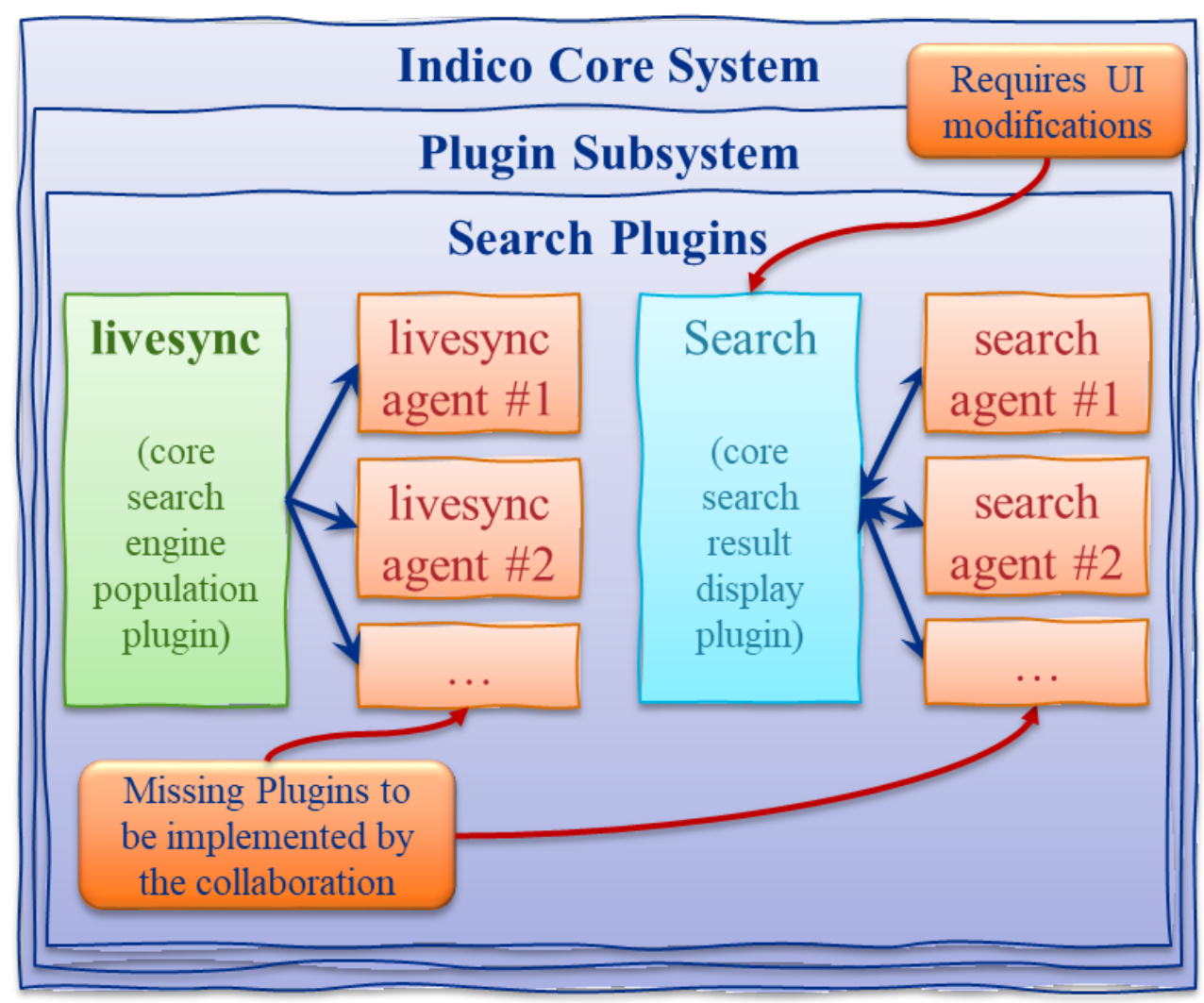

Figure 1. Indico code architecture 


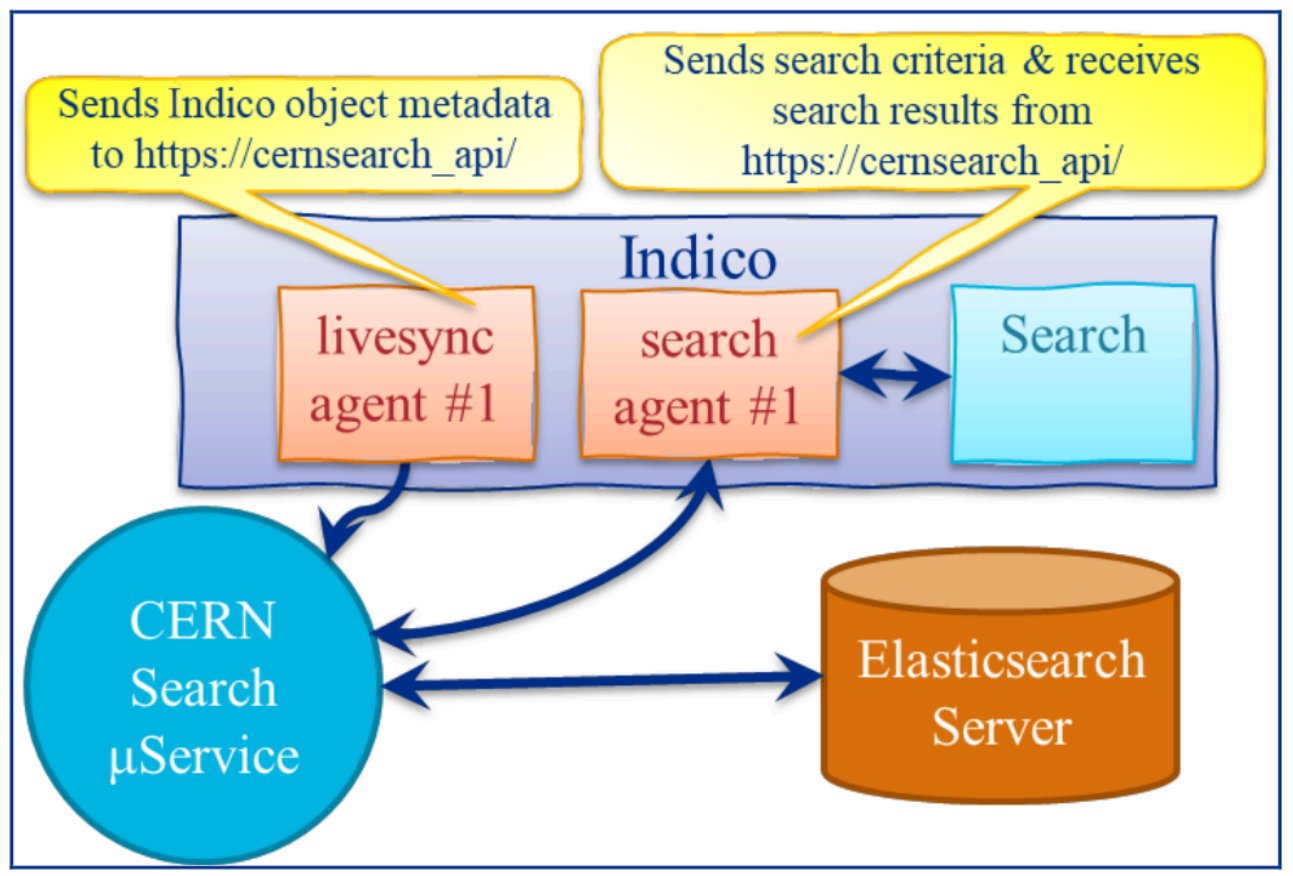

Figure 2. Indico proposed search system architecture

\section{Implementation challenges}

Indico v2.x moved away from the ZODB to PostgreSQL and almost the entire Indico code was re-written and restructured. For an outside developer, any familiarity with the previous versions' code is not useful. The new version provides an easier plugin development method, but the trade-off is that one now needs to understand all the internals of the new Indico plugin system, as well as the interface with the core plugins and the core code, along with numerous new Python packages.

The agreed upon development of the search plugin agents requires interfacing with the CERN Search microservice which is very new, and documentation has thus far been targeted for CERN's internal use. The deployment of the microservice through dockercompose also proved to be more challenging than expected. The CERN provided docker-compose.yml that deploys the necessary docker containers for the microservice, worked as expected, but the configuration to populate the Elasticsearch mappings was not that obvious.

Apart from the coding difficulties, the FNAL and BNL developers worked for a fraction of their time on the Indico project and were not familiar with the new Python packages.

\section{Implementation status}

The existing core livesync plugin will be used as is and will be communicating with the new livesync agent, LiveSync_Json, that was built by the collaboration. A new core search plugin, that includes the user interface, has been implemented by CERN and is in place. 


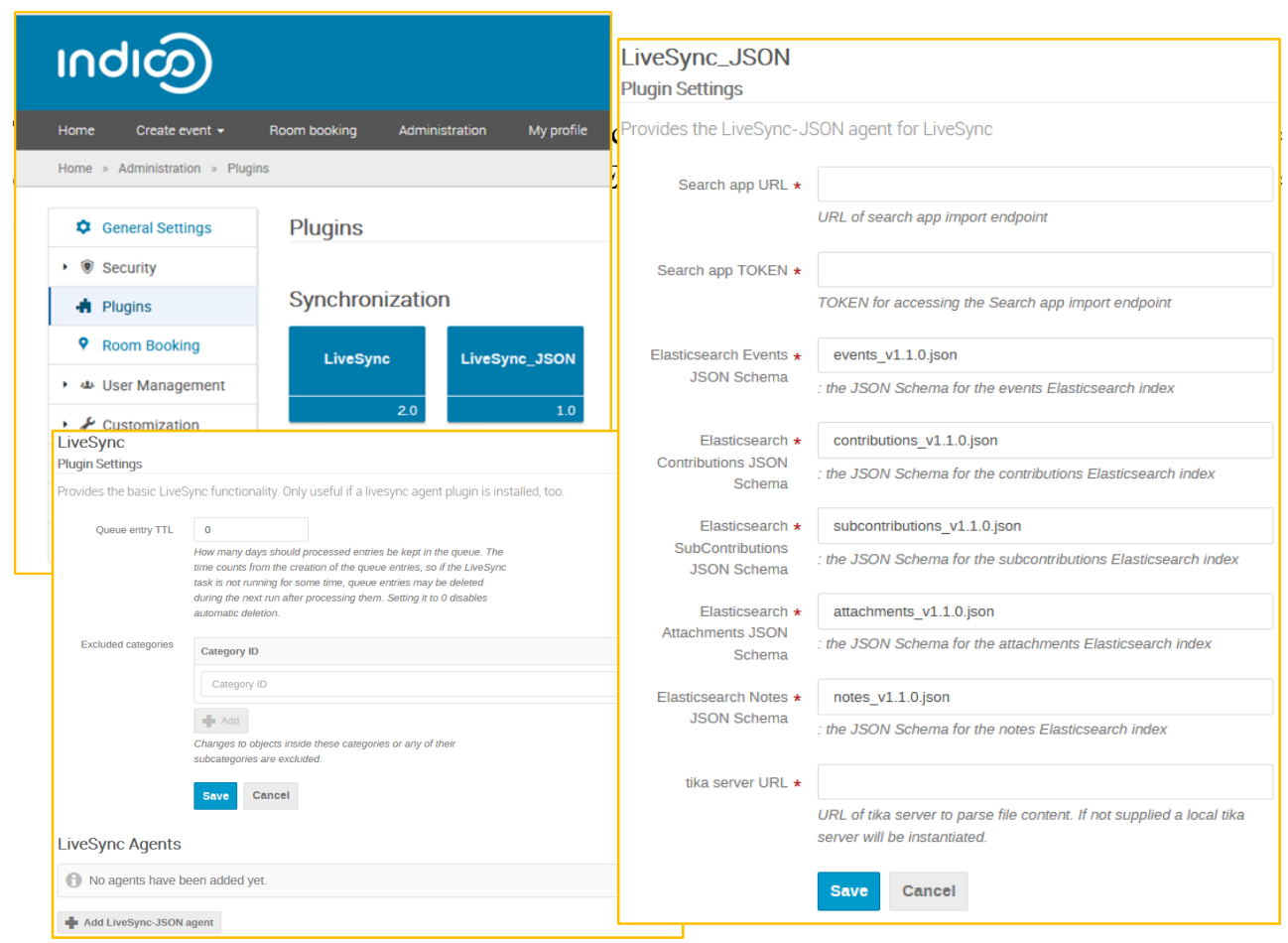

Figure 3. Livesync plugins setup pages

\subsection{Indico livesync agent plugin configuration}

Figure 3 contains screenshots of the livesync and LiveSync_Json configuration.

The plugins are listed under the Plugins administration page and each is configured separately. The livesync plugin configuration is simple and not significantly changed from previous versions of Indico. In contrast, the LiveSync_Json configuration is now much more elaborate. It requires not only the URL and authorization token needed to communicate with the CERN Search microservice, but also the Elasticsearch schema file names, used in the initialization of the microservice for building the json structures needed by the search engine.

\subsection{Indico search user interface}

Figure 4 contains screenshots of Indico's new search user interface as implemented by CERN for Indico v2.x.

The search results are paginated using the plugin's configuration of search results number per page and categorized by Categories, Events, Contributions, Sub-Contributions, Notes, Attachments. The search results can be further filtered by Speaker Name and/or Affiliation. 


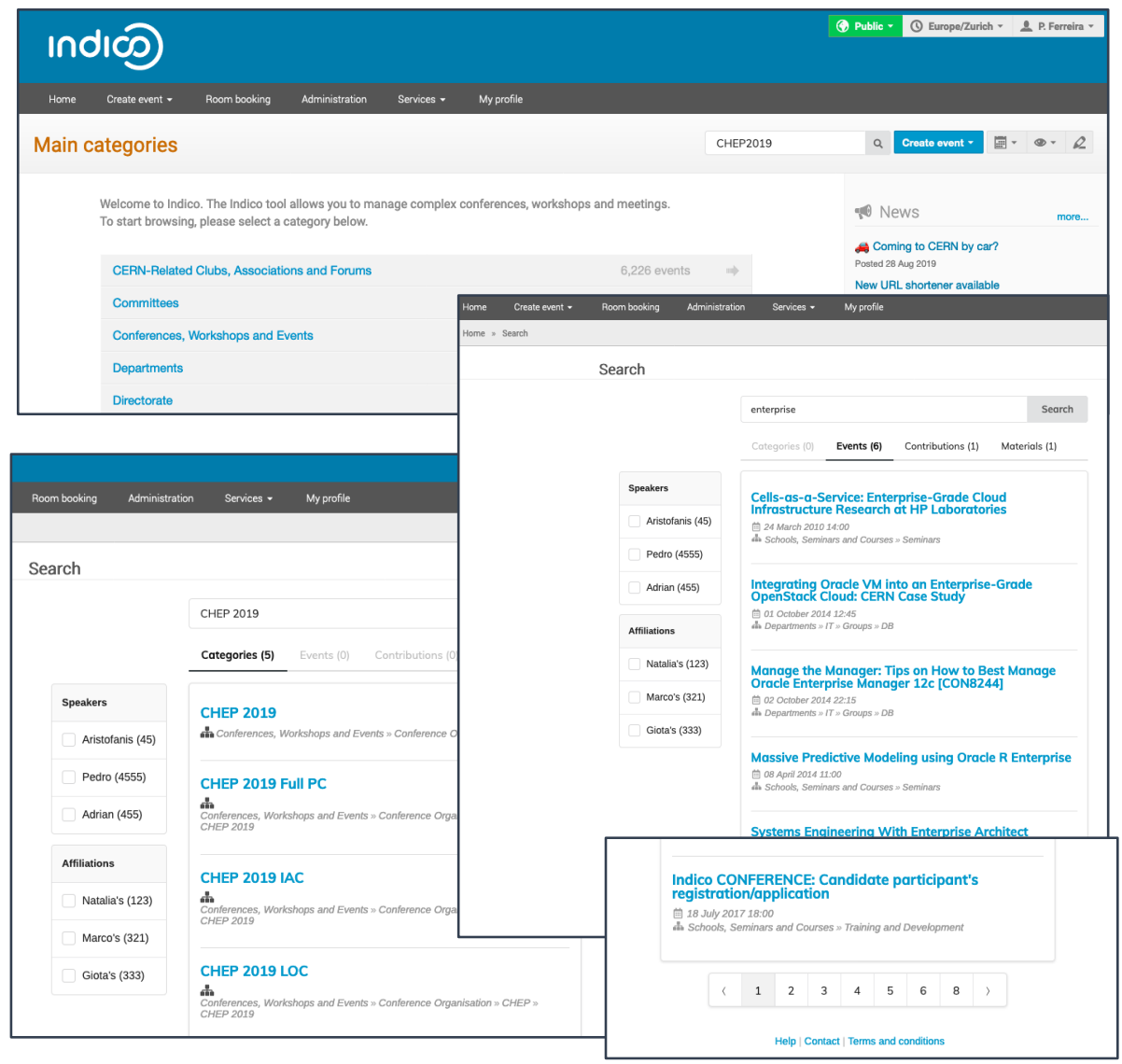

Figure 4. Indico's search UI and search results display

\section{Future Development}

All the agent plugins developed by the collaboration will be integrated into Indico and CERN will take ownership. These plugins will be available to the Indico community to use along with the CERN Search microservice.

Further development may include improved resilience and recovery for the livesync agent; extensions to the search UI, if needed; and last, but not least, improved developer documentation and deployment instructions for non-CERN environments.

\section{References}

1. https:/getindico.io/

2. https://docs.getindico.io/en/latest/

3. https://docs.getindico.io/en/latest/plugins/

4. https://iopscience.iop.org/article/10.1088/1742-6596/664/5/052012

5. https://iopscience.iop.org/article/10.1088/1742-6596/898/10/102017 